"This is the peer reviewed version of the following article: Experts, conflicts of interest and reputation for ability., International J ournal of Economic Theory 10(2):219-233 06 May 2014 which has been published in final form at https://doi.org/10.1111/ijet.12037 This article may be used for non-commercial purposes in accordance with Wiley Terms and Conditions for Self-Archiving." 


\title{
Experts, Conflicts of Interest, and Reputation for
}

\author{
Ability* $^{*}$ \\ Filippo Pavesi ${ }^{\mathrm{a}, \dagger}$, Massimo Scotti ${ }^{\mathrm{b}, \sharp}$ \\ ${ }^{a}$ Department of Economics, University of Milan-Bicocca. \\ ${ }^{\mathrm{b}}$ Economics Discipline Group, University of Technology, Sydney.
}

March 2013

\begin{abstract}
We analyze a model of cheap talk in which an expert that faces a conflict of interest with a decision maker is concerned about establishing a reputation for having accurate information. In this environment, the incentive of the expert to establish a reputation for competence has a non-monotonic effect on the degree of information revelation. An increase in reputation above a certain threshold always makes truthful revelation more difficult to achieve. This is driven by the fact that experts with greater reputation for
\end{abstract}

*We would like to thank Pierpaolo Battigalli, Paolo Colla, Amil Dasgupta, Giovanna Iannantuoni, Marco Ottaviani, Andrea Prat, Jean-Charles Rochet, and Guido Tabellini. We also thank Stefano Bonini and Abigail Brown, and all participants at the 2012 AEA/ASSA annual meeting in Chicago, NASMES 2011 in St. Louis, the EEA 2010 Congress in Glasgow, the Asset 2010 Conference in Alicante, and the SERC 2009 in Singapore for helpful comments. Financial support from Universita' L. Bocconi, the School of Finance and Economics at UTS Sydney, the Department of Economics at University of Milano-Bicocca, and the Department of Public Policy at CEU Budapest is gratefully acknowledged. All errors remain our own.

${ }^{\dagger}$ Corresponding author present address: Department of Industrial Engineering, University of Parma, Viale G.P. Usberti 181/A, I43124 Parma, Italy. Phone: +39 328 3255281; Email: filippo.pavesi@unibocconi.it.

${ }^{\ddagger}$ Economics Discipline Group, University of Technology, Sydney, PO Box 123 Broadway NSW 2007 Australia. Phone: +61 2 95147776; Email: massimo.scotti@uts.edu.au. 
ability can more easily sway the beliefs of decision makers in a desired direction. Thus, higher levels of reputation exacerbate the incentives of biased experts to misreport their private information. Decision makers may therefore be better off consulting less reputable experts when conflicts are more pronounced.

Keywords: Experts; Reputation; Cheap Talk; Conflicts of Interest; Information Transmission.

JEL Classification: C72, D82, D83

\section{Introduction}

There are several economic and political settings in which an expert that is called on to provide information to a decision maker faces an intrinsic conflict of interest. In many such cases, it is plausible to presume that the decision maker is aware of this conflict, at least to some extent. For example, many investors are likely to have a good understanding of the fact that financial analysts have incentives to provide biased reports. ${ }^{1}$ Similarly, in the political arena, the electoral body is likely to know that government agencies have reasons to bias their macroeconomic forecasts towards those that favor politicians. ${ }^{2}$ A standard argument is that the concern of an expert about establishing a reputation for being competent should mitigate this conflict. ${ }^{3}$

The central question that we address in this paper is how reputation for ability and more in general the perceived quality of an expert's information affect the communication process

\footnotetext{
${ }^{1}$ See for example Michaeli and Womack (1999) and Barber et al. (2006, 2007) showing that affiliated analysts have an optimism bias resulting from their involvement in the investment banking activity of their brokerage house.

${ }^{2}$ Weatherford (1987), Alesina and Roubini (1997) and Carlsen (1999) document that incumbent governments generally prefer agencies that are more inclined to provide optimistic forecasts. In these cases, the conflict of interest originates from the ability of the executive branch to sanction agencies that fail to act in its interest by proposing budget cuts, disposing of executives or even advocating termination of the agency.

${ }^{3}$ See for example Mikhail et al. (1999), Hong and Kubik (2003) and Fang and Yasuda (2009) for the case of financial analysts. Heclo (1975), Rourke (1992), Carpenter (2001), Wilson (1989), Bendor et al. (1985) and Banks and Weingast (1992) document the disciplining role of reputation and career concerns in the political arena.
} 
in a context in which the expert's bias is commonly known. We find that in the presence of conflicts of interest, decision makers are not necessarily better off when they consult more reputable experts (i.e., experts with a better expected quality of information). Indeed, we show that it may be optimal for a decision maker to consult a less reputable expert precisely when incentives are less aligned.

We derive these conclusions in a model of cheap talk in which an expert privately observes a (binary) signal about a (binary) state of the world, and subsequently makes a cheap talk report to a decision maker. ${ }^{4}$ The accuracy of the signal depends on the ability of the expert, which is unknown both to the expert and the decision maker. The decision maker observes the report and updates her belief about the state. Once the state has been publicly revealed, the decision maker uses the report to also update her belief about the ability of the expert. To capture the presence of conflicts of interest and the expert's reputational concern for ability, we assume that the payoff of the expert is increasing both in the decision maker's belief that the state is high and in the decision maker's belief about the ability of the expert. Thus, when deciding which report to make, the expert trades off the reputational reward of providing a correct report against the benefit of using his credibility to sway the receiver's beliefs about the state in the desired direction. The payoff function of the expert is assumed to be common knowledge. This implies that the decision maker is aware of the expert's bias.

A distinctive feature of our model is that an increase in the level of initial reputation has a non-monotonic effect on the expert's incentives to truthfully reveal his information. In particular, beyond a certain threshold, any increase in initial reputation always increases the expert's incentives to sway the beliefs of the decision maker towards the desired (high) state. This is in contrast with the case of no conflicts of interest, where an increase in the level of initial reputation always has a positive effect on the expert's incentives to truthfully reveal his information. Intuitively, as reputation becomes sufficiently high, there is less scope for reputation acquisition and reputation becomes less effective in mitigating the expert's

\footnotetext{
${ }^{4}$ We use male pronouns for the expert and female pronouns for the decision maker.
} 
bias. ${ }^{5}$ At the same time, as the reputation of an expert increases, the decision maker assigns more weight to his advice. This in turn provides a biased expert with a higher incentive to misreport.

Thus, when an expert has an explicit conflict of interest, his incentives to misreport increase endogenously with his level of reputation. This has two implications. First, for higher and higher values of initial reputation, truthful revelation occurs for lower and lower beliefs about the state of the world. This is so because when beliefs about the state of the world are strongly pessimistic, a positive message has little effect in inducing decision makers to believe that things are actually going well, thereby attenuating the strong incentives of a highly reputable expert to misreport. Second, ceteris paribus, the distortionary incentives provided by reputation may induce a more reputable expert to lie when a less reputable one does not, implying that in the presence of conflicts of interest decision makers are not necessarily better off when they consult more reputable experts. In particular, we show that an increase in conflicts of interest tends to exacerbate the perverse incentives of reputation. Indeed, we find that as the bias becomes more severe, hiring experts with greater reputation becomes less and less convenient.

Finally, we show that in the presence of conflicts of interest and reputational concerns for ability, truthful revelation becomes possible only when public information is rather contrary to the state towards which the expert wishes to sway the beliefs of the decision maker. For example, in our binary model, an expert with a strong bias towards the high state will report truthfully only when the prior probability of the high state is relatively small. This result, which again arises from the interaction between the expert's conflict of interest and his reputational concerns, suggests that a biased expert should be consulted over issues for which public consensus is polarized around a belief that is opposite to the one that the expert

\footnotetext{
${ }^{5}$ This effect is consistent with the reputational incentives identified by Holmström (1999) in a model of effort provision, where managers exert more effort in the initial stages of their career, when uncertainty on their ability is higher and the scope for reputation acquisition is greater.
} 
would like to induce.

Our paper is related to two main strands of the literature on sender-receiver models of information transmission. The first strand analyzes information transmission in the case in which senders' and receivers' preferences are misaligned (Crawford and Sobel 1982, Sobel 1985; Benabou and Laroque 1992; Morris 2001). The second deals with experts that do not have explicit conflicts of interest with decision makers and are exclusively concerned about establishing a reputation for having accurate information (Ottaviani and Sorensen 2001, 2006; Trueman 1994).

A standard result of the first strand is that only noisy information can be credibly transmitted if the expert and the decision maker have conflicting preferences. In particular, the more biased the expert is, the noisier the information revealed (Crawford and Sobel 1982). Starting with Sobel (1985), this literature has analyzed games of cheap-talk in which there is uncertainty on the preferences of the expert, and the expert can establish a reputation for being unbiased (Benabou and Laroque 1992; Morris 2001). In particular, Morris (2001) highlights a potentially distortionary effect of reputation by showing that an advisor with preferences aligned with those of the decision maker may in fact distort his private information in order to build a reputation for being unbiased. ${ }^{6}$ In our model, the preferences of the expert are assumed to be common knowledge and uncertainty is about the forecasting ability of the expert. The issue we address is whether experts with a higher reputation for competence are more likely to credibly transmit their information when it is well known that they are biased.

Our paper is closely related to Ottaviani and Sorensen $(2001,2006)$. They study information reporting by privately informed experts who are solely motivated by the desire to be perceived as competent, and show that honesty is impossible under very general conditions. In their model, the amount of information that is credibly transmitted is always increasing

\footnotetext{
${ }^{6}$ Ely and Valimaki (2003) obtain a result in the spirit of Morris (2001) in a principal-agent model whitout cheap-talk.
} 
in the quality of the expert's information. By introducing conflicts of interest in a setting with reputation for ability, we show that greater quality of information is not necessarily associated with less misreporting. In some respect, our work is complementary to Bourjade and Jullien (2011) who also consider the case of a biased expert with a reputational concern for competence, but in a setting in which the expert has hard information. They consider strategic concealment of private information while we analyze the issue of misrepresentation of information.

The remainder of the paper is organized as follows. In Section 2, we introduce the general setup of the model. Section 3 characterizes the most informative equilibrium and analyzes the conditions under which truthtelling is possible, highlighting the incentives that lead experts to deviate from truthtelling. Section 4 examines the relationship between the reputation of an expert and his credibility, and Section 5 studies how changes in the intensity of conflicts of interest affect this relationship. Section 6 concludes.

\section{The Model}

An expert is called upon to provide information to a decision maker (DM) who has to make a forecast about the state of world. The state of the world $\omega$ is either low or high, i.e., $\omega \in\{0,1\}$, and all players hold the same prior belief $\theta$ that the state is 1 . At the beginning of the game, the expert observes a private and non-verifiable signal $s \in\left\{s_{0}, s_{1}\right\}$ about the true state, and its accuracy depends on the expert's ability $t$. We assume that the expert is either good or bad, i.e., $t \in\{g, b\}$, and that ability affects the accuracy of the signal as follows:

$$
\begin{aligned}
& \operatorname{Pr}\left(s_{1} \mid t=g, \omega=1\right)=\operatorname{Pr}\left(s_{0} \mid t=g, \omega=0\right)=p, p \in(1 / 2,1), \\
& \operatorname{Pr}\left(s_{1} \mid t=b, \omega=1\right)=\operatorname{Pr}\left(s_{0} \mid t=b, \omega=0\right)=z, z \in(1 / 2, p] .
\end{aligned}
$$


Therefore, both expert types can count on an informative (yet imperfect) signal, with the good type having a more accurate signal than a bad type. ${ }^{7}$ We assume that neither the expert nor the decision maker know the expert's type, and all players hold the same prior belief $\alpha$ that the expert is good. We interpret $\alpha$ as the prior reputation for ability of the expert.

After observing the signal, the expert chooses to release a report to the decision maker in the form of a costless binary message $m \in\left\{m_{0}, m_{1}\right\}$. The decision maker observes message $m$ and chooses an action $x \in \mathbb{R}$. Finally, the true state of the world is revealed and together with the message of the expert is used by the decision maker to revise her beliefs about the expert's ability. ${ }^{8}$ We denote with $\widehat{\alpha}_{\omega, m} \equiv \operatorname{Pr}(t=g \mid \omega, m)$, the decision maker's posterior belief that the expert is good upon observing state $\omega$ and message $m$. We interpret $\widehat{\alpha}_{\omega, m}$ as the new level of reputation for ability acquired by the expert at the end of the game.

The decision maker's utility depends on the state of the world $\omega$ and her action $x$. As is standard in the literature, we assume that the decision maker's utility is given by the quadratic loss function $-(x-\omega)^{2}$. This implies that the action that maximizes the expected utility of the decision maker is equal to the probability she assigns to the state of the world being 1 . Given that message $m$ was sent by the expert with prior reputation $\alpha$, we denote with $\widehat{\theta}_{\alpha, m} \equiv \operatorname{Pr}(\omega=1 \mid m)$ the decision maker's interim belief (i.e., prior to the realization of the state of the world) that the state of the world is 1 . Thus, in equilibrium the decision maker' optimal action is $x=\widehat{\theta}_{\alpha, m}$. As we will see, in any equilibrium in which some information is transmitted, the higher the reputation of the expert, the more the decision maker will trust the message sent. The subscript $\alpha$ highlights this relationship.

To model the expert's concern about establishing a reputation for being a valuable provider of information and the contemporaneous existence of conflicts of interest, we con-

\footnotetext{
${ }^{7}$ All the results also when the bad type has an uninformative signal (i.e., $z=1 / 2$ ).

${ }^{8}$ As in Ottaviani and Sorensen (2006), the fact that the decision maker performs the task of forecasting the expert's ability can be justified by the implicit assumption that receivers are rewarded for accurately forcasting the ability of the expert.
} 
struct a game where the payoff of the expert depends positively on the decision maker's action $x$ as well as on the posterior belief $\widehat{\alpha}_{\omega, m}$, as follows:

$$
\pi(m)=k x+(1-k) \widehat{\alpha}_{\omega, m} \text { with } k \in[0,1]
$$

Since in equilibrium $x=\widehat{\theta}_{\alpha, m}$, the expert's payoff can be rewritten as:

$$
\pi(m)=k \widehat{\theta}_{\alpha, m}+(1-k) \widehat{\alpha}_{\omega, m} \text { with } k \in[0,1]
$$

The component $\widehat{\alpha}_{\omega, m}$ captures the concern of the expert to be perceived as having accurate information. ${ }^{9}$ The component $\widehat{\theta}_{\alpha, m}$ gives the expert an incentive to inflate the decision maker's belief that the state is 1 , and thus creates a conflict of interest with the decision maker. ${ }^{10}$ Finally, the parameter $k \in[0,1]$ weighs these two components and can be seen as a measure of the severity of conflicts of interest. The structure and the parameters of the game (with the sole exception of the expert's signal) are common knowledge. ${ }^{11}$

Notice that interpreting 1 and 0 respectively as favorable and unfavorable states for the decision maker, the model represents the over-optimism bias that has been discussed both in the finance literature on sell side analysts and in the political science literature on government agencies' forecasts. ${ }^{12}$ For the sake of exposition, in the remainder of the paper we will adopt this interpretation and refer to the expert's bias as to the over-optimism bias.

\footnotetext{
${ }^{9}$ This reduced form to account for reputational concerns is widely adopted in studies that model the reputation of experts and managers (see for example Sharfstein and Stein (1990), Ottaviani and Sorensen (2006) and Gentzkow and Shapiro (2006)).

${ }^{10}$ Formally this game falls in the class of psychological games since the sender's payoff depends on the receiver's belief (Battigalli and Dufwenberg 2009).

${ }^{11} \mathrm{It}$ is worth noticing that since also $k$ is common knowledge, we do not address the case in which receivers are uncertain about the incentives of the expert. See Sobel (1985), Benabou and Laroque (1992), and Morgan and Stocken (2003) for a formal analysis of the case in which there is uncertainty about the expert's preferences.

${ }^{12}$ Assuming that the expert has an interest in inflating the receiver's belief about the state being 1 , is without loss of generality. Our setup is well suited for analyzing a more general setting, in which the expert has an incentive to manipulate the receiver's beliefs in a desired direction.
} 


\section{Equilibrium Analysis}

In this section, we analyze the incentives of an expert to truthfully report his information and characterize the most informative equilibrium. ${ }^{13}$

At the moment of sending message $m$, the true state of the world is unknown to the expert. The expert uses his signal $s$ to compute the expected impact of message $m$ on his reputation, as follows:

$$
E\left(\widehat{\alpha}_{\omega, m} \mid s\right)=\operatorname{Pr}(\omega=1 \mid s) \widehat{\alpha}_{1, m}+\operatorname{Pr}(\omega=0 \mid s) \widehat{\alpha}_{0, m} .
$$

Therefore, the expected payoff of the expert from sending message $m$ reads:

$$
E(\pi(m) \mid s)=k \widehat{\theta}_{\alpha, m}+(1-k) E\left(\widehat{\alpha}_{\omega, m} \mid s\right)
$$

Before analyzing the incentives of an expert to truthfully report his information, it is convenient to gain an intuition of the tensions involved in the reporting decision. In any equilibrium where some information is transmitted we have that $\widehat{\theta}_{\alpha, m_{1}}>\widehat{\theta}_{\alpha, m_{0}} \cdot{ }^{14}$ This introduces an incentive to report message $m_{1}$ and represents a threat to truthtelling whenever signal $s_{0}$ is received. In fact, the presence of reputational concerns counterbalances this over-optimism bias. As long as $k \in(0,1)$, the expert has to trade off the temptation of sending $m_{1}$ with the negative effects that this message might have on his reputation in case the message turns out to be incorrect.

The equilibrium concept we use is that of Perfect Bayesian Equilibrium (PBE). The expert will truthfully report signal $s$ if and only if the expected payoff of truthtelling is greater than the payoff of reporting a message that is different from the signal received.

\footnotetext{
${ }^{13}$ Our model presents the well-known problem of equilibrium multiplicity that is common to any cheap-talk game. A babbling equilibrium where all messages are taken to be meaningless and ignored always exists.

${ }^{14}$ Since the expert's signals are informative, in any equilibrium where signals are truthfully reported with some positive probability, the messages of the expert contain some information.
} 
Thus, a truthtelling equilibrium exists if and only if for every $s \in\left\{s_{0}, s_{1}\right\}, E(\pi(m=s) \mid s) \geq$ $E(\pi(m \neq s) \mid s)$, or equivalently:

$$
\begin{aligned}
& k \widehat{\theta}_{\alpha, m_{0}}+(1-k) E\left(\widehat{\alpha}_{\omega, m_{0}} \mid s_{0}\right) \geq k \widehat{\theta}_{\alpha, m_{1}}+(1-k) E\left(\widehat{\alpha}_{\omega, m_{1}} \mid s_{0}\right), \\
& k \widehat{\theta}_{\alpha, m_{1}}+(1-k) E\left(\widehat{\alpha}_{\omega, m_{1}} \mid s_{1}\right) \geq k \widehat{\theta}_{\alpha, m_{0}}+(1-k) E\left(\widehat{\alpha}_{\omega, m_{0}} \mid s_{1}\right) .
\end{aligned}
$$

In a truthtelling equilibrium, posterior reputation takes on only two possible values, which we denote with $\underline{\alpha}$ and $\bar{\alpha}$, where:

$$
\begin{aligned}
& \underline{\alpha} \equiv \widehat{\alpha}_{0, m_{1}}=\widehat{\alpha}_{1, m_{0}}, \\
& \bar{\alpha} \equiv \widehat{\alpha}_{1, m_{1}}=\widehat{\alpha}_{0, m_{0}},
\end{aligned}
$$

with $\bar{\alpha}>\alpha>\underline{\alpha} .{ }^{15}$ Making a correct evaluation increases the expert's reputation from its initial level $\alpha$ to the higher level $\bar{\alpha}$. Making a wrong evaluation decreases the expert's reputation from $\alpha$ to the lower level $\underline{\alpha}$. In the rest of the paper we denote $(\bar{\alpha}-\underline{\alpha})$ as the reputational reward of being recognized as a good expert. This allows us to write conditions (6) and (7) in the following way:

$$
\begin{aligned}
& k\left(\widehat{\theta}_{\alpha, m_{1}}-\widehat{\theta}_{\alpha, m_{0}}\right) \leq(1-k)(\bar{\alpha}-\underline{\alpha})\left(1-2 \operatorname{Pr}\left(\omega=1 \mid s_{0}\right)\right), \\
& k\left(\widehat{\theta}_{\alpha, m_{1}}-\widehat{\theta}_{\alpha, m_{0}}\right) \geq(1-k)(\bar{\alpha}-\underline{\alpha})\left(1-2 \operatorname{Pr}\left(\omega=1 \mid s_{1}\right)\right) .
\end{aligned}
$$

For each of the above conditions, we refer to the left hand side as the benefit of providing a high message, and to the right hand side as the expected reputational gain of sending a low message. Notice that the right hand side of (10) represents the expected reputational gain of truthtelling when receiving a low signal, while the right hand side of (11) represents the expected reputational gain of misreporting when receiving a high signal.

\footnotetext{
${ }^{15}$ We show this result in the Appendix.
} 
It is straightforward to show that when reputation does not play any role (i.e., when $k=1$ ), condition (10) is never satisfied and a truthtelling equilibrium never exists. We now establish that whenever the expert is concerned about reputation some information can be transmitted.

Lemma 1 Independently of whether the expert faces a conflict of interest (i.e., for any $k \in[0,1)$ ), there always exists a non empty interval $[\underline{\theta}, \bar{\theta}]$, with $0<\underline{\theta}<\bar{\theta}<1$ such that the most informative equilibrium is: (i) separating (i.e. fully revealing) for $\theta \in[\underline{\theta}, \bar{\theta}]$ and (ii) pooling (i.e. uninformative) for $\theta \notin[\underline{\theta}, \bar{\theta}]$.

(Proof: see Appendix)

When $\theta$ is relatively extreme, the expert believes that any contrarian signal he receives is likely to be incorrect. Being afraid that ex-post incorrect messages may negatively affect his reputation, he disregards his private information and reports the signal that is more likely to be correct ex-post. This is the conservative behavior highlighted by Ottaviani and Sorensen $(2001,2006)$ for the case in which the expert does not have any partisan bias and is solely concerned about his reputation.

There is a simple reason why this behavior persists in our context with conflicts of interest. When $\theta$ is very low (high), the decision maker expects the state to be 0 (1) regardless of the message sent. As a result, the net gain from inflating the beliefs of the the decision maker by sending $m_{1}$ instead of a $m_{0}$ is very small (i.e., the LHSs of conditions (10) and (11) are close to zero) and the choice of the expert is mainly driven by reputational concerns.

The previous finding highlights that when public opinion is polarized, conflicts of interest are less relevant. No matter how strong is the conflict (i.e., how large is $k$ ), the bias-driven incentives to misreport are small since the expert's ability to sway the decision maker's beliefs in a desired direction is limited. As we show in sections 4 and 5 , the presence of conflicts of interest is not innocuous with respect to other relevant dimensions of the problem that we are analyzing. 


\section{The Effects of Reputation on Credibility}

In this section, we address the following question. Assuming that the decision maker could choose among a pool of experts with different levels of reputation, would she always choose the expert with the highest reputation? As it will soon become clear, in order to answer this question it is sufficient to study how the truthtelling region $[\underline{\theta}, \bar{\theta}]$ is affected by a change in prior reputation $\alpha$. It is useful to begin our analysis by considering the benchmark case in which there are no conflicts of interest (i.e., $k=0$ ).

Remark 1 In the absence of conflicts of interest, the truthtelling region $[\underline{\theta}, \bar{\theta}]$ is symmetrically centered around $\theta=\frac{1}{2}$ and, ceteris paribus, expands monotonically as $\alpha$ increases.

\section{(Proof: see Appendix)}

This result implies that a decision maker can never be worse off by consulting an expert with a higher reputation. If an expert with a certain level of reputation truthfully reveals his information, then any expert with a higher level of reputation will also report truthfully and will do so with a higher expected precision of his signal.

The situation is quite different in the presence of conflicts of interest (i.e., $k \in(0,1))$ since a variation in prior reputation has a non-monotonic effect on the credibility region. Figure 1 compares the case of conflicts of interest (left panel) with the case of no conflicts of interest (right panel). As opposed to the case without conflicts, when conflicts are present, a further increase in prior reputation above a certain threshold makes the truthtelling region shrink, and the thresholds $\underline{\theta}$ and $\bar{\theta}$ shift downwards towards lower values of $\theta$. Therefore, truthtelling holds for a smaller range of values on the state of the world, and this range of values is skewed towards zero. An implication of this result is that for higher and higher values of initial reputation, in order for the expert to truthfully reveal his information, it must be less and less likely that the state of the world is high (i.e., that $\omega=1$ ). This result is summarized in the following proposition: 
Proposition 1 In the presence of conflicts of interest, there always exist $0<\alpha^{*}<\alpha^{* *}<1$ such that: a) For $\alpha \in\left(0, \alpha^{*}\right)$, an improvement in initial reputation $\alpha$ increases the truthtelling thresholds $\underline{\theta}$ and $\bar{\theta}$, and expands the truthtelling region; b) For $\alpha \in\left(\alpha^{* *}, 1\right)$, an improvement in initial reputation $\alpha$ decreases the truthtelling thresholds $\underline{\theta}$ and $\bar{\theta}$, and shrinks the truthtelling region.

\section{(Proof: see Appendix)}

To gain the intuition behind proposition 1, consider the truthtelling conditions (10) and (11). First, note that the net benefit of sending a high report, $\widehat{\theta}_{\alpha, m_{1}}-\widehat{\theta}_{\alpha, m_{0}}$ is increasing in the level of prior reputation $\alpha$. This is so because an expert with a higher level of reputation is expected to have more accurate signals. As a consequence, in any informative equilibrium, the messages of this expert will have a greater impact on the decision maker's beliefs about the state. Then, note that the net reputational reward of being recognized as a good expert, $\bar{\alpha}-\underline{\alpha}$ is strictly concave in $\alpha$, and progressively shrinks to zero as $\alpha$ approaches either zero or one. This occurs because the scope for reputation acquisition is greater, the higher the uncertainty about the expert's ability and becomes negligible when the uncertainty about ability is very low. ${ }^{16}$ The interaction between these two effects determines the way in which a variation in $\alpha$ affects the truthtelling region. As $\alpha$ increases above a certain threshold, the net reputational reward of being recognized as a good expert (i.e., $\bar{\alpha}-\underline{\alpha}$ ) starts to shrink, while the net benefit of sending a high message (i.e., $\widehat{\theta}_{\alpha, m_{1}}-\widehat{\theta}_{\alpha, m_{0}}$ ) keeps growing larger. Eventually, the incentives of the expert to sway the beliefs of the decision maker in favor of the high state grow larger. In order to counter these incentives and preserve truthtelling, $\theta$ must decrease. Indeed, as the probability that the state is high gets smaller, the expected reputational reward of sending a low message gets larger.

This effect intensifies as $\alpha$ approaches one, in which case the two thresholds $\underline{\theta}$ and $\bar{\theta}$ approach zero and the truthtelling region progressively shrinks becoming an empty set. A

\footnotetext{
${ }^{16}$ This is consistent with Holmstrom (1999), in which the reputational incentives for an agent to provide effort are positively related to the level of uncertainty on the agent's ability.
} 
similar reasoning suggests that an increase in $\alpha$ has a positive effect on the size of the truthtelling region whenever the initial level of reputation is below a certain threshold.

An important consequence of introducing conflicts of interest in a setting in which experts are concerned about their reputation for ability is that consulting experts of higher reputation is not necessarily an optimal choice for decision makers. Combining Lemma 1 and Proposition 1, we obtain the following proposition:

Proposition 2 Suppose the DM could choose an expert among those with different prior reputation $\alpha \in[0,1]$. For every prior on the state of the world $\theta \in(0,1)$, there always exists a level of reputation $\alpha^{\prime} \in[0,1)$, such that the DM would never be better off by choosing an expert with prior reputation $\alpha>\alpha^{\prime}$.

\section{(Proof: see Appendix)}

Unlike in the absence of conflicts of interest, the distortionary incentives provided by reputation may induce a more reputable expert to lie when a less reputable expert would not. This suggests that in the presence of conflicts of interest, decision makers are not necessarily better off consulting more reputable experts. To see this point, consider the following example. Assume there are two experts with reputation $\alpha_{0}$ and $\alpha_{1}$ respectively, where $\alpha_{0}<\alpha^{\prime \prime}<\alpha_{1}$. Suppose that the prior on the state of the world is $\theta=\bar{\theta}\left(\alpha^{\prime \prime}\right)$. The expert with high reputation has a truthtelling threshold $\bar{\theta}\left(\alpha_{1}\right)<\bar{\theta}\left(\alpha^{\prime \prime}\right)$ and will not provide any information while the expert with low reputation has a truthtelling threshold $\bar{\theta}\left(\alpha_{0}\right)>\bar{\theta}\left(\alpha^{\prime \prime}\right)$ and will provide valuable information. Thus, if the prior on the state of the world is $\theta=\bar{\theta}\left(\alpha^{\prime \prime}\right)$ and the DM could choose which expert to hire, she would choose the expert with lower reputation. 


\section{Variations in Conflicts of Interest}

We conclude our analysis by considering how the magnitude of conflicts of interest represented by $k$, may affect the results presented in the previous section.

In order to examine the relationship between conflicts of interest and the nature of the truthtelling region, we analyze how variations in $k$ affect the truthtelling thresholds $\underline{\theta}$ and $\bar{\theta}$. The following proposition highlights that when the prior on the state of the world is rather pessimistic, experts with a greater conflict of interest may be more likely to tell the truth than experts with a lower bias.

Proposition 3 As conflicts of interest increase, the truthtelling region shifts downwards (i.e. both $\underline{\theta}$ and $\bar{\theta}$ are decreasing in $k$ )

(Proof: see Appendix)

We know from remark 1 that in the absence of a bias (i.e., $k=0$ ), the truthtelling region

is centered around $\theta=\frac{1}{2}$. Proposition 3 suggests that as conflicts of interest become more severe, the truthtelling region progressively shifts towards values of the prior on the state of the world that are closer to zero. The reason for this result is straightforward. Suppose that $k=k^{\prime}$ and that the expert has received $s_{0}$. We know that in this case the expert reports the low message for all values of $\theta$ in the region $\left(0, \bar{\theta}_{k^{\prime}}\right]$, with $\bar{\theta}_{k^{\prime}}$ denoting the threshold value of $\theta$ at which the expert is indifferent between the high and the low message. If $k$ increases above $k^{\prime}$, the optimistic bias of the expert increases too, and the expert's indifference at $\bar{\theta}_{k^{\prime}}$ is broken in favour of the high message. Similarly, if the expert has received $s_{1}$, he reports the high message for values of $\theta$ in the region $\left[\underline{\theta}_{k^{\prime}}, 1\right)$, where $\underline{\theta}_{k^{\prime}}$ denotes the threshold value of $\theta$ at which the expert is indifferent between the high and the low message. Again, as $k$ increases and the expert's optimistic bias becomes stronger, the indifference at $\underline{\theta}_{k^{\prime}}$ will be broken in favor of the high message.

In general, the previous analysis suggests that when an expert is biased, truthful revelation occurs when public information is rather contrary to the state towards which the 
expert wishes to sway public opinion. Therefore, in situations in which public information is polarized around a certain belief, consulting an expert with a contrary bias may be more valuable than consulting an unbiased expert.

As we have seen in section 4 , in the presence of conflicts of interest, the distortionary incentives provided by reputation may induce a more reputable expert to lie when a less reputable expert would not. We now establish that an increase in conflicts of interest tends to exacerbate the perverse incentives of reputation. Indeed, as the bias becomes more severe (i.e., as $k$ increases), hiring experts with greater reputation becomes less and less convenient. This result is highlighted in the following proposition:

Proposition 4 For any prior on the state of the world $\theta$ such that an expert truthfully reveals his information, an increase in $k$ strictly reduces $\alpha^{\prime}$.

\section{(Proof: see Appendix)}

Decision makers may therefore be better off consulting less reputable experts precisely when incentives are less aligned. Moreover, reputation becomes less effective in mitigating conflicts of interest exactly when it is more needed.

\section{Conclusions}

Conflicts of interest are relevant in many economic settings in which an expert with privileged information is called upon to provide information to an uninformed decision maker. In this paper, we focused on the trade-off that biased experts typically face between the short-term benefits of providing a biased report and the long-term rewards of establishing a reputation for having accurate information.

We find that the interaction between reputation and conflicts of interest plays an important role in shaping the incentives of experts. Reputation for ability allows for some 
information transmission even when decision makers know that an expert is biased. However, reputation has a non-monotonic effect on information transmission. In particular, when the expert's reputation rises above a critical threshold, the expert is more likely to misreport. Therefore decision makers may be better off choosing experts of lower reputation. This occurs because a report sent by an expert with an established reputation for ability, has a greater impact on the beliefs of the decision maker. Accordingly, a highly reputable expert has greater incentives to cash in on reputation by swaying the beliefs of decision makers in the desired direction. Moreover, when conflicts of interest are more intense, reputation is less effective in inducing experts to reveal their information. Therefore, decision makers should consult experts with a lower reputation for ability when their preferences are less aligned.

A suggested avenue for future research is to gather further insight on the role of both ability and preferences in jointly determining the reputation of experts. In particular, a relevant question involves understanding in which circumstances decision makers may be better off consulting experts that have similar preferences, rather than those that have more accurate information. Capturing how these elements may affect the credibility of an expert through the reputational channel represents an open issue. 


\section{Appendix}

Proof of Lemma 1. We divide the proof of Lemma 1 into the following three Parts:

A Truthtelling exists for $[\bar{\theta}, \underline{\theta}]$.

B No informative equilibria exist outside $[\underline{\theta}, \bar{\theta}]$.

C Babbling equilibria always exist.

\section{Part A - Truthtelling exists for $[\bar{\theta}, \underline{\theta}]$}

In a truthtelling equilibrium, the values of posterior reputation and the values of the posterior beliefs about the state read respectively:

$$
\begin{gathered}
\widehat{\alpha}_{\omega, m} \equiv \operatorname{Pr}(t=g \mid \omega, m)= \begin{cases}\frac{\alpha p}{q} \equiv \bar{\alpha} & \text { for }\left(\omega=1, m=m_{1}\right),\left(\omega=0, m=m_{0}\right) . \\
\frac{\alpha(1-p)}{1-q} \equiv \underline{\alpha} & \text { for }\left(\omega=1, m=m_{0}\right),\left(\omega=0, m=m_{1}\right) .\end{cases} \\
\widehat{\theta}_{\alpha, m_{1}}=\operatorname{Pr}\left(\omega=1 \mid s_{1}\right)=\frac{\theta q}{\theta q+(1-\theta)(1-q)} \\
\widehat{\theta}_{\alpha, m_{0}}=\operatorname{Pr}\left(\omega=1 \mid s_{0}\right)=\frac{\theta(1-q)}{\theta(1-q)+(1-\theta) q}
\end{gathered}
$$

where $q \equiv \alpha p+(1-\alpha) z$.

These results allow us to rewrite truthtelling conditions (10) and (11) as follows:

$$
\begin{aligned}
\frac{k \theta(1-\theta)(2 q-1)}{(\theta+q-2 q \theta)(1+2 q \theta-\theta-q)} & \leq \frac{(1-k) \alpha(p-q)(q-\theta)}{q(1-q)(\theta+q-2 q \theta)} \\
\frac{k \theta(1-\theta)(2 q-1)}{(\theta+q-2 q \theta)(1+2 q \theta-\theta-q)} & \geq \frac{(1-k) \alpha(p-q)(1-\theta-q)}{q(1-q)(1+2 \theta q-\theta-q)}
\end{aligned}
$$


Consider condition (A1). Since $\frac{1}{2} \leq z<q<p<1$ and $\theta \in(0,1)$, we have that $\theta+q-2 q \theta>0$. Thus, (A1) is equivalent to:

$$
\frac{k \theta(1-\theta)(2 q-1)}{1+2 q \theta-\theta-q} \leq \frac{(1-k) \alpha(p-q)(q-\theta)}{q(1-q)}
$$

Let $l(\theta) \equiv \frac{k \theta(1-\theta)(2 q-1)}{1+2 q \theta-\theta-q}$ and $r(\theta) \equiv \frac{(1-k) \alpha(p-q)(q-\theta)}{q(1-q)}$. Note that for every $\alpha \in(0,1), k \in$ $[0,1), z \in\left(\frac{1}{2}, p\right)$ and $p \in(z, 1)$ :

Property (a): $l(0)=0<r(0), l(1)=0>r(1)$;

Property (b): $r(\theta)$ is a negatively sloped straight line;

Property (c): $l(\theta)$ is non-negative, continuous, and strictly concave for $\theta \in(0,1)$.

Properties (a), (b) and (c) imply that there exists a unique $\bar{\theta} \in(0,1)$ such that (A3) is satisfied for any $\theta<\bar{\theta}$.

Focusing on condition (A2) and following the same line of reasoning above, one can prove that there exists a unique $\underline{\theta} \in(0,1)$ such that (A2) is satisfied for any $\theta>\underline{\theta}$.

We now show that $\bar{\theta}>\underline{\theta}$. Note that:

Property (d): The LHSs of (A1) and (A2) have the same expression. This expression is equal to zero at $\theta=0,1$. It is strictly positive and strictly concave in $\theta$ for $\theta \in(0,1)$.

Property(e): The expression of the $R H S$ of (A1) is strictly larger than the expression of the $R H S$ of (A2). Both expressions are strictly decreasing in $\theta$.

Together with the uniqueness of $\underline{\theta}$ and $\bar{\theta}$, properties (d) and (e) imply that $\bar{\theta}>\underline{\theta}$.

We conclude step A by establishing a property that we will use later, namely:

Property $(f): \bar{\theta} \leq q$.

To see that Property (f) holds, note that $\bar{\theta}$ is the value of $\theta$ at which (A1) holds with equality. Now note that $R H S$ of (A1) is strictly negative for values of $\theta$ strictly larger than q. This result, together with properties (d) and (e) above, imply that equality can occur only at a value of $\theta$ smaller than or equal to $q$.

\section{Part B - No informative equilibria exist outside $[\underline{\theta}, \bar{\theta}]$}


An informative equilibria exists as long as it satisfies the following two conditions:

$$
\begin{aligned}
& k \widehat{\theta}_{\alpha, m_{1}}-k \widehat{\theta}_{\alpha, m_{0}} \leq(1-k) E\left(\widehat{\alpha}_{\omega, m_{0}} \mid s_{0}\right)-(1-k) E\left(\widehat{\alpha}_{\omega, m_{1}} \mid s_{0}\right), \\
& k \widehat{\theta}_{\alpha, m_{1}}-k \widehat{\theta}_{\alpha, m_{0}} \geq(1-k) E\left(\widehat{\alpha}_{\omega, m_{0}} \mid s_{1}\right)-(1-k) E\left(\widehat{\alpha}_{\omega, m_{1}} \mid s_{1}\right)
\end{aligned}
$$

For simplicity, let us rewrite these conditions using the following short-hand notation:

$$
\begin{aligned}
& L H S \leq R H S\left(s_{0}\right) \\
& L H S \geq R H S\left(s_{1}\right)
\end{aligned}
$$

Aside truthtelling, the following (mixed-strategy) informative equilibria are to be considered:

PP1: The expert truthfully reports $m_{1}$ after signal $s_{1}\left(R H S\left(s_{1}\right)<L H S\right)$, while he reports $m_{0}$ with probability $u_{0,0}$ and $m_{1}$ with probability $1-u_{0,0}$ after signal $s_{0}\left(R H S\left(s_{0}\right)=\right.$ $L H S)$

PP0: The expert truthfully reports $m_{0}$ after signal $s_{0}\left(R H S\left(s_{0}\right)>L H S\right)$, while he reports $m_{1}$ with probability $u_{1,1}$ and $m_{0}$ with probability $1-u_{1,1}$ after signal $s_{1}$ (LHS= RHS $\left.\left(s_{1}\right)\right)$.

PP: The expert follows a mixed strategy both after $s_{0}$ and $s_{1}$. He reports $m_{1}$ with probability $u_{1,1}$ and $m_{0}$ with probability $1-u_{1,1}$ after signal $s_{1}\left(R H S\left(s_{0}\right)=L H S\right)$; and he reports $m_{0}$ with probability $u_{0,0}$ and $m_{1}$ with probability $1-u_{0,0}$ after signal $s_{0}\left(R H S\left(s_{1}\right)=\right.$ $L H S)$; where $u_{0,0} \neq u_{1,1}$.

Note that since signals are informative, $\operatorname{Pr}\left(\omega=1 \mid s_{1}\right)>\operatorname{Pr}\left(\omega=1 \mid s_{0}\right)$ and $\operatorname{Pr}(\omega=$ $\left.0 \mid s_{0}\right)>\operatorname{Pr}\left(\omega=0 \mid s_{1}\right)$; Furthermore, whenever messages contain some information, $\widehat{\alpha}_{\omega=m} \geq \widehat{\alpha}_{\omega \neq m}$. Given this, it it follows that $R H S\left(s_{0}\right)>R H S\left(s_{1}\right)$ for any $\theta$ so that:

$$
R H S\left(s_{0}\right)-L H S>R H S\left(s_{1}\right)-L H S \text { for any } \theta
$$


We show that none of the three equilibria above exists for $\theta>\bar{\theta}$ (a similar reasoning applies for $\theta<\underline{\theta})$.

We first show that PP1 does not exist. Suppose that the expert receives $s_{0}$. The expert will follow the equilibrium strategy of sending $m_{0}$ with probability $u_{0,0}$ and $m_{1}$ with probability $1-u_{0,0}$ if and only if condition (A4) holds with equality, that is:

$$
k \widehat{\theta}_{\alpha, m_{0}}+(1-k) E\left(\widehat{\alpha}_{\omega, m_{0}} \mid s_{0}\right)=k \widehat{\theta}_{\alpha, m_{1}}+(1-k) E\left(\widehat{\alpha}_{\omega, m_{1}} \mid s_{0}\right)
$$

Calculating the equilibrium values of $\widehat{\theta}_{\alpha, m_{0}}, \widehat{\theta}_{\alpha, m_{1}}, \widehat{\alpha}_{\omega, m_{0}}$ and $\widehat{\alpha}_{\omega, m_{1}}$, condition (A9) reads as follows:

$$
\frac{k(1-\theta) \theta(2 q-1)}{(q+\theta-2 \theta q)\left(1-u_{0,0}(q-2 \theta q+\theta)\right)}=\frac{\alpha(1-k)(p-q)\left(1-2 \theta+\theta u_{0,0}-u_{0,0}(1-q)\right)}{\left(1-u_{0,0} q\right)\left(1-u_{0,0}(1-q)\right)(q-2 \theta q+\theta)}
$$

This equality can equivalently be written as:

$$
\frac{k(1-\theta) \theta(2 q-1)\left(1-u_{0,0} q\right)}{\left(1-u_{0,0}(q-2 \theta q+\theta)\right)}=\frac{\alpha(1-k)(p-q)\left(1-2 \theta+\theta u_{0,0}-u_{0,0}(1-q)\right)}{\left(1-u_{0,0}(1-q)\right)}
$$

We have to show that there does not exist any $u_{0,0} \in(0,1)$ such that (A10) is satisfied when $\theta \in(\bar{\theta}, 1)$. Since we know from Property (f) in Step A that $\bar{\theta} \leq q$, it is convenient to consider the following two cases:

- $\theta \in(q, 1)$. Note that when $\theta \in(q, 1)$, the $L H S$ of (A10) is positive, while the $R H S$ is strictly negative because $1-2 \theta+\theta u-u(1-q)<0$. Thus, (A10) can never be satisfied for $\theta \in(q, 1)$.

- $\theta \in(\bar{\theta}, q]$. Note that the LHS and RHS of (A10) satisfy the following properties 
respectively:

$$
\begin{aligned}
\frac{\partial L H S}{\partial u_{0,0}} & =-\frac{k(1-\theta) \theta(2 q-1)^{2}}{\left(u_{0,0}(\theta+q-2 \theta q)-1\right)^{2}}<0 \text { for } \theta \in(0,1) \\
\frac{\partial^{2} L H S}{\partial u_{0,0}^{2}} & =\frac{2 k(1-\theta)(\theta-2 q \theta)^{2}(\theta+q-2 \theta q)}{\left(u_{0,0}(\theta+q-2 \theta q)-1\right)^{3}}<0 \text { for } \theta \in(0, q] \\
\frac{\partial R H S}{\partial u_{0,0}} & =\frac{\theta(\alpha(1-k)(p-q)(2 q-1)}{\left(1-u_{0,0}+q u_{0,0}\right)^{2}}>0 \text { for } \theta \in(0,1) \\
\frac{\partial^{2} R H S}{\partial u_{0,0}^{2}} & =\frac{2 \alpha \theta(1-k)(p-q)(1-q)(2 q-1)}{\left(1-u_{0,0}+q u_{0,0}\right)^{3}}>0 \text { for } \theta \in(0,1)
\end{aligned}
$$

In words, the $R H S$ is strictly increasing and strictly convex in $u_{0,0}$ for $\theta \in(0,1)$, while the $L H S$ is strictly decreasing and strictly concave in $u_{0,0}$ for $\theta \in(0, q]$. Properties (A11) to (A14) imply that when $\theta \in(0, q]$, if a $u_{0,0}$ exists such that condition (A10) is satisfied, it must be unique. Now, we know that at $u_{0,0}=1$ (i.e. when there is truthtelling) the following two facts hold true: a) Condition (A10) is satisfied for $\theta=\bar{\theta}$; b) The LHS of condition (A10) is strictly greater than the $R H S$ for $\theta \in(\bar{\theta}, q]$. But then, by properties (A11) to (A14) we have that for all $\theta \in(\bar{\theta}, q]$, there does not exist any $u_{0,0} \in(0,1)$ such that (A10) can be satisfied.

We now show that PP0 does not exist. We have just shown that for every $\theta>$ $\bar{\theta}$, PP1 does not exist.. In particular, $R H S\left(s_{0}\right)-L H S<0$ By (A8), this also implies that $R H S\left(s_{1}\right)-L H S<0$. Therefore if PP1 never exists also PP0 never exists (because PP0 requires that $\left.R H S\left(s_{1}\right)-L H S=0\right)$.

We finally show that $\mathbf{P P}$ does not exist. PP requires both (A6) and (A7) to be satisfied with equality, which in turn implies that $R H S\left(s_{0}\right)=R H S\left(s_{1}\right)$. This violates condition (A8).

\section{Part C - Babbling Equilibria always exist}

We conclude the proof of lemma 1 by showing that a babbling equilibrium where the expert sends $m_{1}$ with probability $\pi$ and $m_{0}$ with probability $1-\pi$ irrespectively of the signal 
observed always exists. In this case all messages are taken to be meaningless and ignored: $\widehat{\theta}_{\alpha, m}=\theta$ for any $m \in\left\{m_{0}, m_{1}\right\}$, and $\widehat{\alpha}_{\omega, m}=\alpha$ for any $\omega \in\{0,1\}$ and $m \in\left\{m_{0}, m_{1}\right\}$, making the expert indifferent between the two messages.

Proof of Remark 1. When $k=0$, condition (A1) boils down to $0 \leq \alpha(p-q)(\theta-q)$. Thus, when $k=0$, the upper bound of the truthtelling region is $\bar{\theta}_{k=0}=q \equiv \alpha p+(1-\alpha) z$. Similarly, from condition (A2), one can show that when $k=0$ the lower bound of the truthtelling region is $\underline{\theta}_{k=0}=1-q \equiv 1-(\alpha p+(1-\alpha) z)$.

Proof of Proposition 1. Consider condition (A1) and notice that for every $k \in$ $(0,1), z \in\left(\frac{1}{2}, p\right)$ and $p \in(z, 1)$, the following holds true: (i) For $\alpha \rightarrow 0, L H S_{1} \rightarrow$ $\frac{k \theta(2 z-1)(1-\theta)}{(2 z \theta-\theta-z)(2 z \theta-\theta-z+1)}$ and $R H S_{1} \rightarrow 0$; thus, for $\alpha \rightarrow 0, \bar{\theta} \rightarrow 0$; (ii) For $\alpha \rightarrow 1, L H S_{1} \rightarrow$ $\frac{k \theta(2 p-1)(1-\theta)}{(2 p \theta-\theta-p)(2 p \theta-\theta-p+1)}$ and $R H S_{1} \rightarrow 0$; thus, for $\alpha \rightarrow 1: \bar{\theta} \rightarrow 0$. Now, (i) and (ii) imply that for $\alpha \rightarrow 0, \bar{\theta}-\underline{\theta} \rightarrow 0$; A similar argument applies to condition (A2) to show that: (iii) For $\alpha \rightarrow 0, \underline{\theta} \rightarrow 0$; (iv) For $\alpha \rightarrow 1, \underline{\theta} \rightarrow 0$. Now, (iii) and (iv) imply that for $\alpha \rightarrow 1, \bar{\theta}-\underline{\theta} \rightarrow 0$.

Since $\bar{\theta}-\underline{\theta}$ is positive for any value of $\alpha \in(0,1)$, by continuity there exists a value of $\alpha=\alpha^{*} \in(0,1)$ below which $\bar{\theta}-\underline{\theta}$ is increasing in $\alpha$, and a value of $\alpha=\alpha^{* *} \in(0,1)$ above which $\bar{\theta}-\underline{\theta}$ is decreasing in $\alpha$. Note that properties (i) to (iv) above imply that $\alpha^{* *}>\alpha^{*}$.

Proof of Proposition 2. Let $\bar{\theta}(k, \alpha, z, p)$ be the solution to condition (A1). Note that Lemma 1 guarantees that $\bar{\theta}(k, \alpha, z, p)$ is defined for every $\alpha \in(0,1), k \in[0,1), z \in\left(\frac{1}{2}, p\right)$, $p \in(z, 1)$.

Since we are interested in changes in $\alpha$ while keeping all other parameters constant, for the sake of notation, we simply write $\bar{\theta}(\alpha)$.

By Lemma 1 and Proposition 1, we know that $\bar{\theta}(\alpha)$ satisfies the following properties for $k \in(0,1)$ and $\alpha \in\left(\alpha^{* *}, 1\right)$ :

(a) $\bar{\theta}(\alpha)$ is continuous and strictly decreasing in $\alpha$;

(b) $\bar{\theta}\left(\alpha^{* *}\right)<1$ and $\bar{\theta}(1) \rightarrow 0$.

For a given $\theta \in(0,1)$, we define $\alpha^{\prime}(\theta)$ as the value of $\alpha$ such that for every $\alpha \in\left(\alpha^{\prime}(\theta), 1\right]$ 
it holds that $\theta>\bar{\theta}(\alpha)$

In order to prove the proposition we need to show that $\alpha^{\prime}(\theta) \in(0,1)$ for every $\theta \in(0,1)$. We therefore consider the following two cases:

1. $\theta \in\left(0, \bar{\theta}\left(\alpha^{* *}\right)\right)$ - Properties (a) and (b) imply that for any given $\theta \in\left(0, \bar{\theta}\left(\alpha^{* *}\right)\right)$ we have that $\alpha^{\prime}(\theta) \in\left(\alpha^{* *}, 1\right)$.

2. $\theta \in\left(\bar{\theta}\left(\alpha^{* *}\right), 1\right)$ - Property (a) implies that for every $\alpha \in\left(\alpha^{* *}, 1\right)$ we have that $\theta>\bar{\theta}(\alpha)$. This implies that $\alpha^{\prime}(\theta) \leq \alpha^{* *}$.

Proof of Proposition 3. To proof proposition 3, first note that:

$$
\begin{aligned}
& \text { In condition (A1), }\left.\frac{\partial R H S}{\partial \theta}\right|_{\theta=\bar{\theta}}<\left.\frac{\partial L H S}{\partial \theta}\right|_{\theta=\bar{\theta}} ; \\
& \text { In condition (A2), }\left.\frac{\partial R H S}{\partial \theta}\right|_{\theta=\underline{\theta}}<\left.\frac{\partial L H S}{\partial \theta}\right|_{\theta=\underline{\theta}} ;
\end{aligned}
$$

This result is a straightforward consequence of Properties (d) and (e) in the Proof of Lemma 1 together with the existence and uniqueness of $\bar{\theta}$ and $\underline{\theta}$. In words, (A15) says that the RHS of (A1) always intersects the LHS from above, and (A16) says that the same is true for condition (A2).

Now, note that for any $\theta \in(0, q), \frac{\partial L H S}{\partial k}=-\frac{\theta(1-\theta)(2 q-1)}{(2 q \theta-\theta-q)(1+2 q \theta-\theta-q)}>0$ and $\frac{\partial R H S}{\partial k}=$ $-\frac{\alpha(p-q)(\theta-q)}{(1-q) q(2 q \theta-\theta-q)}<0$. Since $\bar{\theta}<q$, (see Property (f) in the Proof of Lemma 1), the previous result together with (A15) and (A16) implies that $\bar{\theta}$ is decreasing in $k$. The same reasoning applies to condition (A2) to show that $\underline{\theta}$ is decreasing in $k$.

Proof of Proposition 4. Let us denote with $\bar{\theta}(k, \alpha)$ the solution to condition (A1). Note that Lemma 1 guarantees that $\bar{\theta}(k, \alpha)$ is defined for every $\alpha \in(0,1), k \in[0,1)$.

By the proof of proposition 1, we know that: 
(a) For every $k \in(0,1)$, there always exists an $\alpha^{* *}(k)$ such that $\bar{\theta}(k, \alpha)$ is strictly decreasing for all $\alpha \in\left(\alpha^{* *}(k), 1\right)$.

By proposition 3, we know that the following property holds true:

(b) For any $k_{0}, k_{1} \in(0,1)$ such that $k_{1}>k_{0}$ and every $\alpha \in(0,1), \bar{\theta}\left(k_{1}, \alpha\right)<\bar{\theta}\left(k_{0}, \alpha\right)$.

Now, let:

- $\alpha^{* *}\left(k_{0}\right)$ be the value of $\alpha^{* *}$ when $k=k_{0}$;

- $\bar{\theta}\left(k_{0}, \alpha^{* *}\left(k_{0}\right)\right.$ be the value of $\bar{\theta}(\alpha, k)$ when $k=k_{0}$ and $\alpha=\alpha^{* *}\left(k_{0}\right)$;

- $\alpha^{\prime}\left(k_{0}, \theta\right)$ and $\alpha^{\prime}\left(k_{1}, \theta\right)$ respectively denote the values of $\alpha^{\prime}(\theta)$ when $k=k_{0}$ and $k=k_{1}$, where $\alpha^{\prime}(\theta)$ is defined in proposition 2 .

Then, by properties (a) and (b), for every $\theta \in\left(0, \bar{\theta}\left(k_{0}, \alpha^{* *}\left(k_{0}\right)\right)\right.$ it must be that $\alpha^{\prime}\left(k_{1}, \theta\right)<$ $\alpha^{\prime}\left(k_{0}, \theta\right)$. 


\section{References}

[1] Alesina, A., Roubini, N., and Cohen, G. D., 1997. Political Cycles and the Macroeconomy. Cambridge, MA: MIT Press.

[2] Banks, J. S., Weingast, B. R., 1989. The Political Control of Bureaucracies under Asymmetric Information. American Journal of Political Science, 36, 509-24.

[3] Barber, B., Lehavy, R., McNichols, M., and Trueman, B., 2006. Buys, holds, and sells: The distribution of investment banks' stock ratings and the implications for the profitability of analysts' recommendations. Journal of Accounting and Economics, 41, 87117.

[4] Barber, B., Lehavy, R., and Trueman, B., 2007. Comparing the stock recommendation performance of investment banks and independent research firms. Journal of Financial Economics, 85, 490-517.

[5] Battigalli, P., Dufwenberg, M., 2009. Dynamic Psychological Games. Journal of Economic Theory, 144, 1-35.

[6] Benabou, R., Laroque, G., 1992. Using Privileged Information to Manipulate Markets: Insiders, Gurus and Credibility. Quarterly Journal of Economics, 107, 921-958.

[7] Bendor, J., Taylor, S., and Van Gaalen, R., 1985. Bureaucratic Expertise versus Legislative Authority: A Model of Deception and Monitoring in Budgeting. American Political Science Review, 70, 1041-1060.

[8] Bourjade, S., Jullien, B., 2011. The roles of reputation and transparency on the behaviour of biased experts. Rand Journal of Economics, 42, 575-594.

[9] Carlsen, F., 1999. Inflation and Elections: Theory and Evidence for Six OECD Economies. Economic Inquiry, 37, 120-135. 
[10] Carpenter, D. P., 2001. The Forging of Bureaucratic Autonomy: Reputations, Networks, and Policy Innovation in Executive Agencies, 1862-1928, Princeton, NJ: Princeton University Press.

[11] Crawford, V. P., Sobel, J., 1982. Strategic Information Transmission. Econometrica, 50, 1431-1451.

[12] Ely, J., Valimaki, J., 2003. Bad Reputation, Quarterly Journal of Economics, 118, 785814.

[13] Fang, L., Yasuda, A., 2009. The Effectiveness of Reputation as a Disciplinary Mechanism in Sell-side Research. Review of Financial Studies, 22, 3735-3777.

[14] Gentzkow, M., Shapiro, J. M., 2006. Media Bias and Reputation. Journal of Political Economy, 114, 280-316.

[15] Heclo, H., 1975. OMB and the Presidency - The Problem of 'Neutral Competence'. The Public Interest, 38, 80-99.

[16] Holmström, B., 1999. Managerial Incentive Problems: A Dynamic Perspective. Review of Economic Studies, 66, 169-182.

[17] Hong, H., Kubik, J.D., 2003. Analyzing the Analysts: Career Concerns and Biased Earnings Forecasts. Journal of Finance, 58, 313-351.

[18] Michaely, R., Womak, K. L., 1999. Conflict of Interest and the Credibility of Underwriter Analyst Recommendations. Review of Financial Studies, 12, 653-686.

[19] Mikhail, M.B., Walther, B.R., and Willis, R.H., 1999. Does forecast accuracy matter to security analysts?. Accounting Review, 74, 185-200.

[20] Morgan, J., Stocken, P. C., 2003. An Analysis of Stock Recommendations. RAND Journal of Economics, 34, 183-203. 
[21] Morris, S., 2001. Political Correctness. Journal of Political Economy, 109, 231-265.

[22] Ottaviani, M., Sorensen, P. N., 2001. Information aggregation in debate: who should speak first?. Journal of Public Economics, 81, 393-421.

[23] Ottaviani, M., Sorensen, P. N., 2006. Reputational Cheap Talk. Rand Journal of Economics, 37, 155-175.

[24] Rourke, F. E., 1992. Responsiveness and Neutral Competence in American Bureaucracy. Public Administration Review, 52, 539-546.

[25] Scharfstein, D. S., Stein, J. C., 1990. Herd Behavior and Investment. American Economic Review, 80, 465-479.

[26] Sobel, J., 1985. A Theory of Credibility. Review of Economic Studies, 52, 557-573.

[27] Trueman, B., 1994. Analyst Forecasts and Herding Behavior. Review of Financial Studies, 7, 97-124.

[28] Weatherford, M. S., 1987. How Does Government Performance Influence Political Support?. Political Behavior, 9, 5-28.

[29] Wilson, J. Q., 1989. Bureaucracy: What Government Agencies Do and Why They Do It. New York: Basic Books. 


\section{Figures}

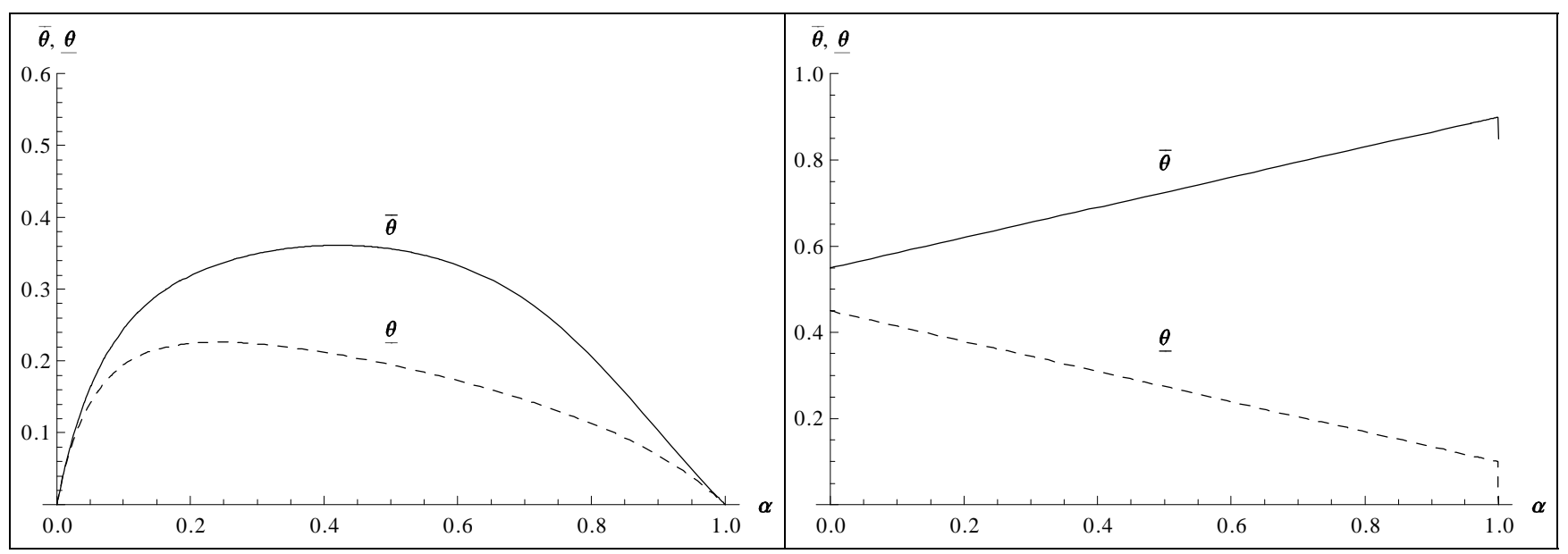

Figure 1: Impact of a variation in $\alpha$ on the size and the position of the truthtelling region in the case of conflicts of interest (left panel) and no conflicts of interest (right panel). 